\title{
ADOLESCENTES ATENDIDOS NUM SERVIÇO PÚBLICO DE URGÊNCIA E EMERGÊNCIA: PERFIL DE
} MORBIDADE E MORTALIDADE.

\section{ADOLESCENTS TAKEN CARE OF IN A PUBLIC SERVICE OF URGENCY AND EMERGENCY: PROFILE OF MORBIDADE AND MORTALITY. \\ ADOLESCENTES TOMADOS CUIDADO ADENTRO DE UN SERVICIO PÚBLICO DE LA URGENCIA Y DE LA EMERGENCIA: PERFIL DE MORBIDAD Y DE LA MORTALIDAD}

\section{Islaine Fernandes Dubuc ${ }^{1}$, Rosângela Aparecidda Pimenta Ferrari ${ }^{2}$}

\begin{abstract}
RESUMO: Trata-se de uma investigação descritiva transversal para caracterizar as causas de morbidade e mortalidade entre adolescentes atendidos no serviço de urgência e emergência de um hospital público. A coleta de dados foi realizada através das fichas de atendimento, nos meses de janeiro, fevereiro e março de 2003, totalizando 2722. A população constituiu-se por adolescentes de 10 a 19 anos de idade, residentes no município. As causas de morbi-mortalidade foram classificadas de acordo com a Classificação Internacional de Doenças (CID10). Mais da metade dos atendimentos é para a população feminina $(54,1 \%)$. As causas de morbidade predominantes foram as doenças infecciosas e parasitárias no sexo feminino $(26,5 \%)$ e lesões e envenenamento e algumas outras conseqüências de causas externas no sexo masculino $(30,5 \%)$. O mês de maior atendimento foi março $(38,4 \%)$. O período da noite foi de maior prevalência (37,6\%). A clínica médica atendeu $63,9 \%$ dos adolescentes. Receberam alta, $84,6 \%$ dos casos e não foram detectados óbitos. Os resultados contribuem para o direcionamento de políticas públicas e efetivação de medidas preventivas, de controle e redução das principais causas de morbidade, que levam a população adolescente a procurar o serviço de pronto socorro.
\end{abstract}

PALAVRAS-CHAVE: Adolescente; Medicina de Emergência; Morbidade; Mortalidade.

ABSTRACT: One is about a transversal descriptive inquiry in the characterize causes of morbidade and mortality between adolescents taken care of in the urgency and emergency service of a public hospital. The collection of data was carried through the attendance fiches, in the January, February and March months of 2003, totalizing 2722. The population consisted of adolescents of 10 the 19 years of age, residents in the city. The morbi-mortality causes had been classified in accordance with the International Classification of Desease (CID-10). More than the half of the taken care is for the feminine population (54.1\%). The predominant causes of morbidade had been the infectious and parasitic illnesses in the feminine sex (26.5\%) and injuries and poisoning and some other consequences of external causes in the masculine sex $(30.5 \%)$. The month of bigger attendance was March (38.4\%). The period of the night was of bigger prevalence (37.6\%). The medical clinic took care of $63.9 \%$ of the adolescents. They had received high, $84.6 \%$ of the cases and had not been detected deaths. The results contribute for the aiming of writ of prevention public politics the specific action, of control and reduction of the main causes of morbidade that take the adolescent population to look the service of ready aid.

KEYWORDS: Adolescent; Medicina de Emergencia; Morbidity; Mortality.

RESUMEN: Uno está sobre una investigación descriptiva transversal que usted caracteriza causas del morbidad y de la mortalidad entre los adolescentes tomados cuidado en del servicio de la urgencia y de la emergencia de un hospital público. La recogida de datos fue llevada a través a través de las fichas de la atención, en los meses de enero, Febrero y marcha de 2003, totalizar 2722. La población consistió en adolescentes de 10 los 19 años de la edad, residentes en la ciudad. La morbimortalidad causa había sido clasificada de acuerdo con la clasificación internacional de Doenças (CID10). Más que la mitad de los tomados cuidado para la población femenina (54.1\%). Las causas predominantes del morbidad habían sido las enfermedades infecciosas y parásitas en el sexo femenino (26.5\%) y lesiones y envenenamiento y algunas otras consecuencias de causas externas en el sexo masculino (30.5\%). El mes de una atención más grande era marcha (38.4\%). El período de la noche estaba de un predominio más grande (37.6\%). La clínica médica tomó el cuidado de $63.9 \%$ de los adolescentes. Habían recibido colmo, $84.6 \%$ de los casos y no habían sido muertes detectadas. Los resultados contribuyen para apuntar de la escritura de la política y del efetivacion políticas públicos de la prevención, del control y de la reducción de las causas principales del morbidad que toman a población adolescente para mirar el servicio de la ayuda lista.

\footnotetext{
${ }^{1}$ Enfermeira do serviço de saúde de Telêmaco Borba, PR, Brasil. ${ }^{2}$ Enfermeira, Docente do Centro de Ciências da Saúde Departamento de Enfermagem - Área da Saúde da Criança e do Adolescente - Universidade Estadual de Londrina, Paraná, Brasil. E-mail: mropimentaferrari@uel.br.
} 
DUBUC, I. F.; FERRARI, R. A. P. Adolescentes atendidos num serviço público de urgência e emergência: perfil de morbidade e mortalidade. Revista Eletrônica de Enfermagem, v. 08, n. $02, \quad$ p. $250 \quad-\quad 258,2006 . \quad$ Disponível em http://www.fen.ufg.br/revista/revista8_2/v8n2a10.htm

PALABRAS CLAVE: Adolescente; Emergency Medicine; Morbilidad; Mortalidad.

\section{INTRODUÇÃO}

Para a Organización Mundial de la Salud OMS (1995) a adolescência constitui-se um processo fundamentalmente biológico de vivências orgânicas. Nesta fase, aceleram-se o desenvolvimento cognitivo e a estruturação da personalidade, abrangendo a etapa da pré-adolescência (faixa etária de 10 a 14 anos), chamada de puberdade na qual ocorre o estirão de crescimento, o aumento rápido das secreções de diversos hormônios e o aparecimento dos caracteres sexuais secundários (maturação sexual) e a adolescência (dos 15 aos 19 anos) que se caracteriza pela desaceleração destes processos.

Os novos conceitos têm destacado os desafios atuais enfrentados pela juventude tais como a violência, a epidemia da aids, a disseminação das doenças sexualmente transmissíveis, o consumo de drogas lícitas e ilícitas, a iniciação sexual precoce, a gravidez não-planejada, entre outros, tornando-os um grupo populacional vulnerável, ou seja, "um grupo social que se encontra em fase de importantes transformações biológicas e mentais, articuladas a um redimensionamento de identidades de papéis sociais" (AYRES \& FRANÇA JR., 1996, p.68)

Mundialmente, na década de 90 , a população de 10 a 19 anos, superou um bilhão. Em 1996, na América Latina e no Caribe havia uma população de 51 milhões de jovens de 15 a 19 anos de idade o que em 2000 passou para 52 milhões. Nos países como México, Honduras, Guatemala e Nicarágua, nos anos de 1988-1998, quase um quarto da população pertencia ao grupo de idade de 15 aos 19 anos e, na Bolívia, República Dominicana, Equador, Panamá e Paraguai, era mais do que a quinta parte da população (CAMACHO HUBNER, 2000).

No Brasil, o censo do Instituto Brasileiro de Geografia e Estatística - IBGE mostrou que no ano de 2000, 46 milhões de brasileiros eram jovens com idade de 10 a 24 anos, distribuídos de forma equivalente entre o sexo feminino e masculino. Em 2005 , os adolescentes representavam $29 \%$ da população total, da qual 17 milhões ocupavam a faixa etária dos 10 aos 14 anos (10,4\%), tendo um acréscimo de 3,3 milhões em relação à geração de 1980 e 16,5 milhões com idade de 15 a 19 anos. Cerca de $70 \%$ dos adolescentes e jovens residiam na cidade e $30 \%$ na área rural (IBGE, 2006).

A população adolescente tenderá a diminuir no Brasil, chegando a $21,6 \%$ do total da população brasileira em 2020. Os pré-adolescentes (10 a 14 anos) poderão passar de 17 milhões em 2000 para 14,5 milhões em 2020. Os adolescentes (15 a 19 anos) serão 17,9 milhões em 2000 e 14,3 milhões em 2020 (BAENINGER, 1999).
A população londrinense, totaliza 447.065 habitantes, com $96,9 \%$ residentes na área urbana. Deste total, 18,7\% (83.550 hab.) são adolescentes, $9,0 \%$ na faixa etária de 10 a 14 anos e $9,7 \%$ na faixa etária de 15 a 19 anos de idade. A população adolescente passou de 20,1\% em 1991 para 18,7\% em 2000 (LONDRINA, 2006).

Este grupo etário representa significativa preocupação para os serviços de saúde pública, e em face desta realidade, o objetivo do presente artigo é caracterizar as causas de morbidade e mortalidade entre adolescentes atendidos num serviço de urgência e emergência de um hospital público do município de Londrina, Paraná.

\section{METODOLOGIA}

A pesquisa constituiu-se um estudo transversal e descritivo acerca dos adolescentes residentes em Londrina e atendidos pelo serviço de urgência e emergência de um hospital público.

A população de estudo se constituiu por adolescentes de 10 a 19 anos de idade residentes no município de Londrina, atendidos no pronto socorro de um hospital público localizado na região norte do município no período de 01 de janeiro a 31 de março do ano de 2004. Os dados foram coletados no período de 20 de março a 30 de agosto de 2004.

Os dados de morbidade foram obtidos no Hospital Dr. Anísio Figueiredo (Hospital Zona Norte HZN) que faz atendimentos de urgência e emergência e internação, que também presta atendimentos aos adolescentes. O HZN é um hospital público, atualmente de nível secundário, com capacidade de 56 leitos, localizado na região norte de Londrina.

A coleta de dados foi realizada através das fichas de atendimento do pronto socorro do hospital. Realizou-se uma busca por fichas de adolescentes entre 10 a 19 anos atendidos no hospital no ano de 2003. Por meios dos livros de registro dessa instituição, selecionaram-se os prontuários pela idade e ano de internação. A partir desta listagem os prontuários foram consultados junto ao SAME do hospital, preenchendo um formulário previamente testado.

Todos os formulários foram codificados manualmente pelas pesquisadoras a fim de verificar o código segundo a Classificação internacional de Doenças, décima revisão (OMS, 2000).

Foi realizada exaustiva verificação de inconsistências através do cruzamento e verificação dos dados.

Os dados foram analisados através do programa Excel versão 2000, utilizando-se freqüência simples e teste qui-quadrado. 
DUBUC, I. F.; FERRARI, R. A. P. Adolescentes atendidos num serviço público de urgência e emergência: perfil de morbidade e mortalidade. Revista Eletrônica de Enfermagem, v. 08, n. $02, \quad$ p. $250 \quad-\quad 258,2006 . \quad$ Disponível em http://www.fen.ufg.br/revista/revista8_2/v8n2a10.htm

Este teste baseia-se nas diferenças entre valores observados e esperados, avaliando se as proporções em cada grupo podem ser consideradas semelhantes ou não. Ou seja, este teste verifica a homogeneidade dos grupos em relação às variáveis categóricas. O nível de significância adotado para este estudo foi de $5 \%$, portanto os valores do $\boldsymbol{p}$ menores que 0,05 ( $p$-valor $<0,05)$ foram apontados como estatisticamente significantes.

Os resultados são apresentados em forma de gráficos e tabelas, em números absolutos e relativos.

Atendendo a Resolução $\mathrm{n}^{\circ}$ 196, de 10 de outubro de 1990, do Conselho Nacional de Saúde, que dispõe sobre as normas regulamentadoras de pesquisa com seres humanos (BRASIL, 1996), foi solicitada a aprovação por escrito do diretor do HZN para o acesso aos prontuários e informações, mediante a garantia de anonimato de todos os sujeitos pesquisados.

O presente estudo foi submetido ao Comitê de Ética em Pesquisa da Universidade Estadual de Londrina, sendo aprovado em 17 de março de 2004.

\section{RESULTADOS E DISCUSSÃO}

Através das analises dos dados do hospital tiveram um total de 74.129 atendimentos realizados no ano de 2003. No presente estudo, foi levantado 2795 prontuários entre os meses de coleta correspondendo a 3,8\% dos adolescentes atendidos no pronto socorro.

Com relação ao sexo, mostra que mais da metade dos atendimentos foram realizados para adolescentes do sexo feminino, enquanto que o sexo masculino representou $45,9 \%$ dos atendimentos. Observou-se que em quase todas as faixas etárias o atendimento foi maior no sexo feminino, porém o sexo masculino superou o sexo feminino na faixa etária dos 12 aos 13 anos de idade.

A predominância do sexo feminino nos serviços de pronto socorro em hospitais gerais se confirma em outro estudo de CASTRO et al. (2002), que obtiveram $60 \%$ de freqüência do sexo feminino.

Entretanto, verificando a distribuição da clientela do pronto socorro de um hospital geral de Itaquaquecetuba, São Paulo, no mês de março de 2002, foram atendidas 288 adolescentes na faixa etária de 10 a 14 anos, sendo a maior procura do sexo masculino (55\%) (YAMADA et al., 2002).

$\mathrm{Em}$ todas as idades dos adolescentes atendidos no serviço, verifica-se que houve predominância entre as idades de 17 a 19 anos, seguidas pelas idades de 16 e 12 anos.

Semelhante a presente casuística, segundo os dados do Datasus (DATASUS, 2006) as internações hospitalares em Londrina no ano de 2003, na faixa etária de 10 a 19 anos somou um total de 3.675. Destas, os atendimentos entre as idades de 15 a 19 anos correspondeu a 2.346 (63,8\%) dos adolescentes e a faixa etária 10 a 14 anos foi de 1.329 (36,1\%).

Observou-se que o número de adolescentes residentes na região Norte foi predominante (94\%).

Tal resultado justifica-se pelo fato do hospital estudado estar localizado na região Norte, e ainda de acordo com o Sistema Informação em Atenção Básica (LONDRINA, 2005), a concentração de adolescentes nesta região (26,3\%) ser maior do que nas encontradas em outras regiões do município.

Analisando-se a Tabela 1, destaca-se a predominância das doenças infecciosas e parasitárias com $696(25,6 \%)$ casos, seguidas pelas lesões, envenenamentos e outras conseqüências de causas externas $(22,8 \%)$ e também pelas doenças do aparelho respiratório $(17,2 \%)$.

Tabela 1 - Distribuição dos adolescentes atendidos no pronto socorro de um hospital público segundo os grupos de causas (capítulos) da Classificação Internacional de Doenças (CID-10), Londrina, janeiro a março, 2003.

\begin{tabular}{|c|c|c|}
\hline Grupo de causas da CID - 10 & $\mathbf{N}^{\circ}$ & $\%$ \\
\hline Cap. I Algumas doenças infecciosas e parasitárias (A00-B99) & 696 & 25,6 \\
\hline Cap. X Doenças do aparelho respiratório (J00-J99) & 468 & 17,2 \\
\hline Cap. XI Doenças do aparelho digestivo (K00-K93) & 82 & 3,0 \\
\hline Cap. XII Doenças da pele e do tecido subcutâneo (L00-L99) & 110 & 4,0 \\
\hline Cap. XIII Doenças do sistema osteomuscular e do tecido conjuntivo (M00-99) & 123 & 4,5 \\
\hline Cap. XIV Doenças do aparelho geniturinário (N00-N99) & 62 & 2,3 \\
\hline $\begin{array}{l}\text { Cap. XVIII Sintomas, sinais e achados anormais de ex. clínicos e de lab., } \\
\text { não classif. em outra parte (R00-R99) }\end{array}$ & 319 & 11,7 \\
\hline $\begin{array}{l}\text { Cap. XIX Lesões, envenenamento e algumas outras conseqüências de } \\
\text { causas externas (S00-T98) }\end{array}$ & 621 & 22,8 \\
\hline Outros & 241 & 8,9 \\
\hline Total & 2722 & 100,0 \\
\hline
\end{tabular}

Em outros estudos, como o de CASTRO et al. (2000), que verificaram a distribuição das internações realizadas no Hospital Geral de Itaim Paulista, no período de maio de 1999 a abril de 2000, observou-se que a doença do aparelho respiratório foi a primeira causa $(21,1 \%)$, seguidas de lesões, envenenamentos e algumas outras conseqüências de causas externas 
DUBUC, I. F.; FERRARI, R. A. P. Adolescentes atendidos num serviço público de urgência e emergência: perfil de morbidade e mortalidade. Revista Eletrônica de Enfermagem, v. 08, $\quad$. $\quad 02, \quad$ p. $250 \quad-\quad 258,2006 . \quad$ Disponível em http://www.fen.ufg.br/revista/revista8 2/v8n2a10.htm

$(13,3 \%)$ e pelas doenças infecciosas e parasitárias $(3,3 \%)$.

Segundo dados do Datasus, em Londrina, no ano de 2003, as internações entre os adolescentes tiveram como principais causas a gravidez, parto e puerpério (29\%), a segunda maior causa foi as doenças do aparelho respiratório (14\%) e a terceira maior causa as lesões, envenenamentos e algumas outras conseqüências de causas externas (12\%) (DATASUS, 2006).

No presente estudo, observou-se grande proporção de doenças infecciosas e parasitárias, entre elas gastrenterites, algumas suspeitas de dengue, sendo que nesta época de acordo com os resultados da pesquisa de TAKEMURA (2003), no município de Londrina ocorria uma epidemia da doença.

Tabela 2 - Distribuição dos adolescentes atendidos no pronto socorro de um hospital público segundo os grupos de causas (capítulos) da Classificação Internacional de Doenças (CID-10) e a faixa etária Londrina, janeiro a março, 2003.
Correlacionando-se as faixas etárias e os grupos de causas, observa-se na Tabela 2, que nas faixas etárias de 10 a 14 anos e 15 a 19 anos, respectivamente $26,4 \%$ e $24,9 \%$, prevaleceram as doenças infecciosas e parasitárias ocasionadas principalmente pelo dengue, gastrenterites e outras doenças infecciosas.

$\mathrm{Na}$ faixa etária de 10 e 14 anos, as lesões, envenenamentos e algumas outras conseqüências de causas externas foram a principal causa $(25,3 \%)$, tais como quedas da própria altura e de bicicleta ocasionando ferimentos, escoriações, entorses e traumas leves. Em segunda colocação, destacaramse as doenças do aparelho respiratório, entre estas as amidalites, doença pulmonar obstrutiva crônica (DPOC), broncopneumonia e asma.

\section{Grupo de causas da CID - 10}

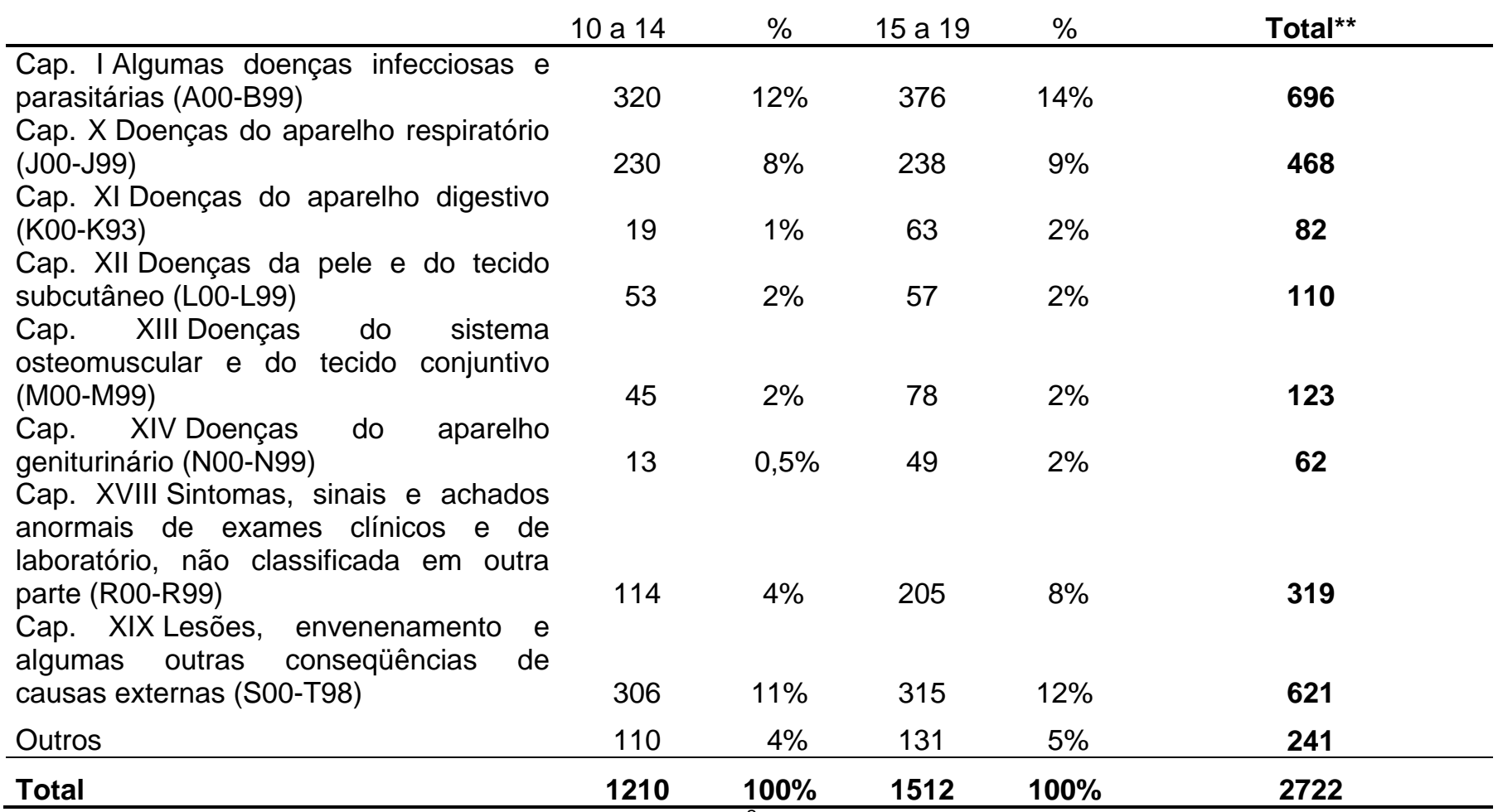

Significativo pelo teste de $\chi^{2}$, ao nível de $1 \%(p \leq 0,01)$.

Em pesquisa realizada por CASTRO et al. (2002), no pronto socorro de um hospital geral, foram atendidos 1268 adolescentes no período de maio de 1999 a abril de 2000 entre as idades de 10 a 14 anos, sendo que a primeira causa de atendimento foram as doenças do aparelho respiratório (25\%), entre estas amidalites e DPOC, seguidas pelas lesões, envenenamentos e algumas outras conseqüências de causas externas $(22,2 \%)$, tais como traumas de mão, tornozelo e punhos, e pelas doenças infecciosas e parasitárias (3,8\%) como as diarréias e gastrenterites.

Em outro estudo, também realizado em um pronto socorro no mês de março de 2002 por YAMADA et al. (2002), foi observado que, na faixa etária dos 10 a 14 anos, as lesões, envenenamentos e algumas outras conseqüências de causas externas foram a primeira causa de atendimento (33,2\%), entre elas fratura de mão e punhos, seguidas de doenças 
DUBUC, I. F.; FERRARI, R. A. P. Adolescentes atendidos num serviço público de urgência e emergência: perfil de morbidade e mortalidade. Revista Eletrônica de Enfermagem, v. 08, $\quad$. $\quad 02, \quad$ p. $250 \quad-\quad 258,2006 . \quad$ Disponível em http://www.fen.ufg.br/revista/revista8_2/v8n2a10.htm

do aparelho respiratório $(18,5 \%)$ tendo como principais causas amidalites e bronquite, e as doenças infecciosas e parasitárias (diarréias e gastrenterites) que corresponderam a $7 \%$ dos atendimentos, representando a quarta maior causa.

Em Londrina, no ano de 2003, ocorreu um total de 3675 internações entre a faixa de 10 a 19 anos, 1329 corresponderam às idades de 10 a 14 anos, tendo como primeira causa gravidez, parto $\mathrm{e}$ puerpério $(43,3 \%)$, seguida das doenças do aparelho respiratório (26,4\%), e lesões, envenenamento e algumas outras conseqüências de causas externas $(11,2 \%)$ e doenças infecciosas e parasitárias $(8,4 \%)$ (DATASUS, 2006).

Através de estudos realizados por ANDRADE \& MELLO JORGE (2000) em Londrina, no primeiro semestre de 1996, pesquisando as vítimas de acidentes de transporte terrestre, verificou-se que houve um número considerável de menores de 18 anos conduzindo veículos automotores, perfazendo um total de 982 vítimas. No presente estudo, entre as causas externas, observou-se uma alta freqüência de acidentes envolvendo ciclistas e motociclistas, além dos atropelamentos.
Verifica-se na Tabela 3, que dentre os 45,9\% atendimentos realizados aos adolescentes do sexo masculino predominou as lesões, envenenamento e algumas outras conseqüências de causas externas $(30,5 \%)$ seguido pelas doenças infecciosas e parasitárias $(24,4 \%)$, e pelas doenças do aparelho respiratório $(17,1 \%)$.

Quanto ao sexo feminino, pode-se observar, ainda na Tabela 3 , que as doenças infecciosas e parasitárias corresponderam a $26,5 \%$ dos atendimentos, em sua grande parte foram causadas por gastrenterite, dengue, e outras infecções, seguido pelas doenças do aparelho respiratório em sua maioria por amidalites e faringites $(17,2 \%)$, enquanto que, as lesões, envenenamento e algumas outras conseqüências de causas externas constituíram-se a terceira maior causa $(16,3 \%)$ muitos devido a traumas, ferimentos e entorses de mão, cotovelo e tornozelo ocorrendo em ambos os sexos, porém o sexo feminino se diferenciou pela prevalência de intoxicação por medicamentos algumas em tentativas suicidas.

Tabela 3 - Distribuição dos adolescentes atendidos no pronto socorro de um hospital público segundo o sexo e os grupos de causas (capítulos) da Classificação Internacional de Doenças (CID-10), Londrina, janeiro a março, 2003.

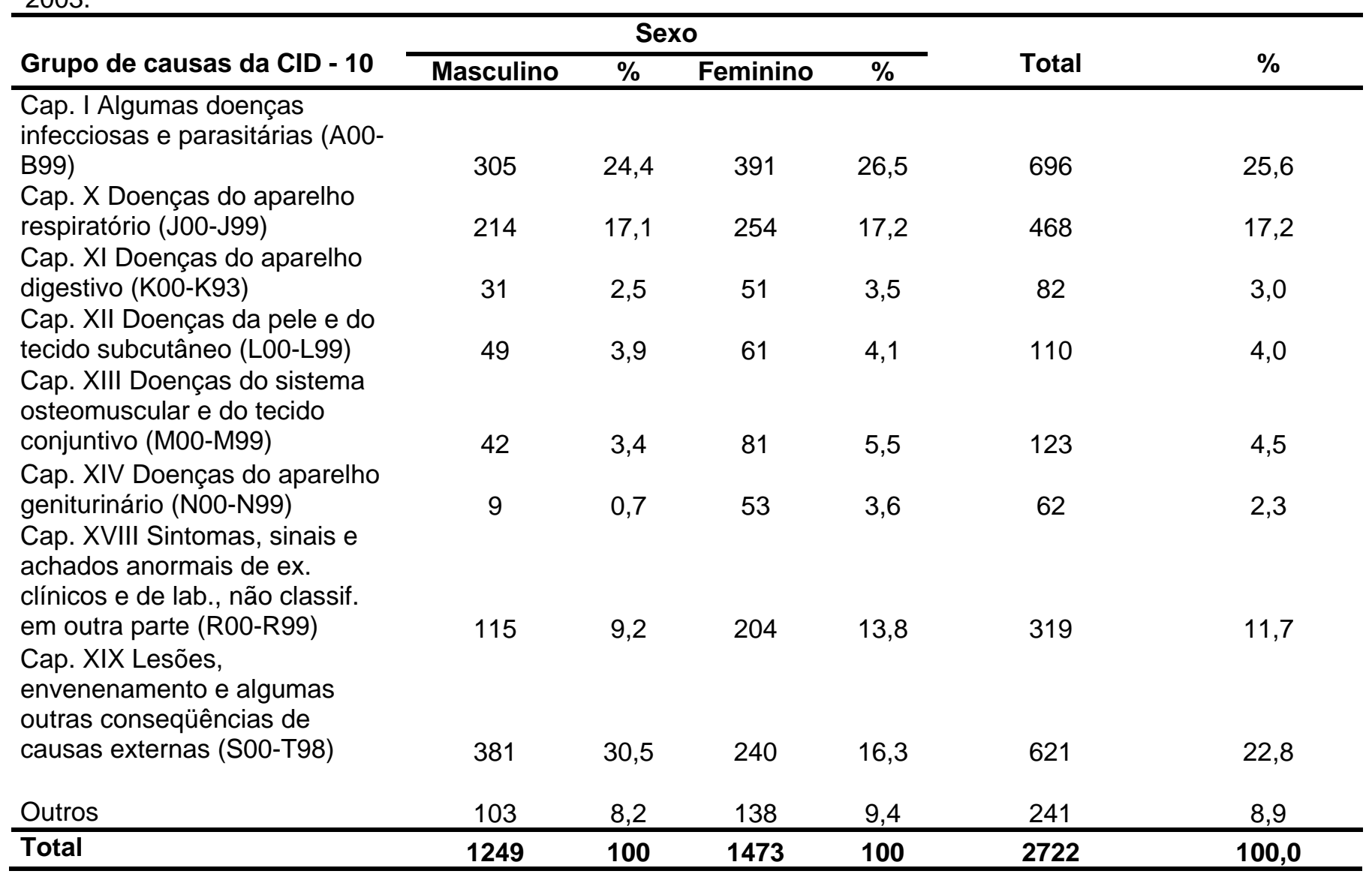

Significativo pelo teste de $\chi 2$, ao nível de $1 \%(p \leq 0,01)$. 
DUBUC, I. F.; FERRARI, R. A. P. Adolescentes atendidos num serviço público de urgência e emergência: perfil de morbidade e mortalidade. Revista Eletrônica de Enfermagem, v. $08, \quad$ n. $\quad 02, \quad$ p. $250 \quad-\quad 258,2006 . \quad$ Disponível em http://www.fen.ufg.br/revista/revista8_2/v8n2a10.htm

Segundo os dados DATASUS (2006), referente ao sexo e motivo de internação no ano de 2003, em Londrina, das 3.675 internações na faixa de 10 a 19 anos a primeira causa entre o sexo masculino foram as lesões, envenenamentos e algumas outras conseqüências de causas externas $(9,2 \%)$, enquanto que a primeira causa para o sexo feminino foi gravidez, parto e puerpério $(29,2 \%)$.

Em vários estudos, verifica-se alta prevalência das lesões, envenenamento e algumas outras conseqüências de causas externas no sexo masculino (ANDRADE \& MELLO JORGE, 2000), principalmente como condutores de veículos, enquanto que 0 sexo feminino se acidentou principalmente como passageiras.

A violência no trânsito também tem contribuído para a alta exposição dos adolescentes, o que pode ser potencializado pelo maior consumo de álcool, drogas, manobras de risco, alta velocidade, entre outros.

A freqüência de lesões, envenenamentos e outras causas externas também pode ser associada a grande tendência às quedas, traumas, perfurações e intoxicações verificadas nas menores faixas etárias (MONTEIRO et al., 2003; LYRA et al., 2002; RIOS et al., 2002).

$\mathrm{Na}$ análise da Tabela 4, observa-se que o mês de março apresentou a maior freqüência $(38,2 \%)$ de atendimentos, sendo a principal causa as doenças infecciosas e parasitárias (31,3\%). Destaca-se que no mês de janeiro (32\%), houve predomínio das lesões, envenenamento e algumas outras conseqüências de causas externas $(23,8 \%)$. No mês de fevereiro, houve um total de $810(29,8 \%)$ de atendimentos tendo como principal causa as doenças infecciosas e parasitárias $(24,0 \%)$.

Tabela 4 - Distribuição dos adolescentes atendidos no pronto socorro de um hospital público em segundo os grupos de causas dos capítulos da CID-10 nos meses de janeiro a março, Londrina, 2003.

Meses

\section{Grupo de causas da CID - 10}

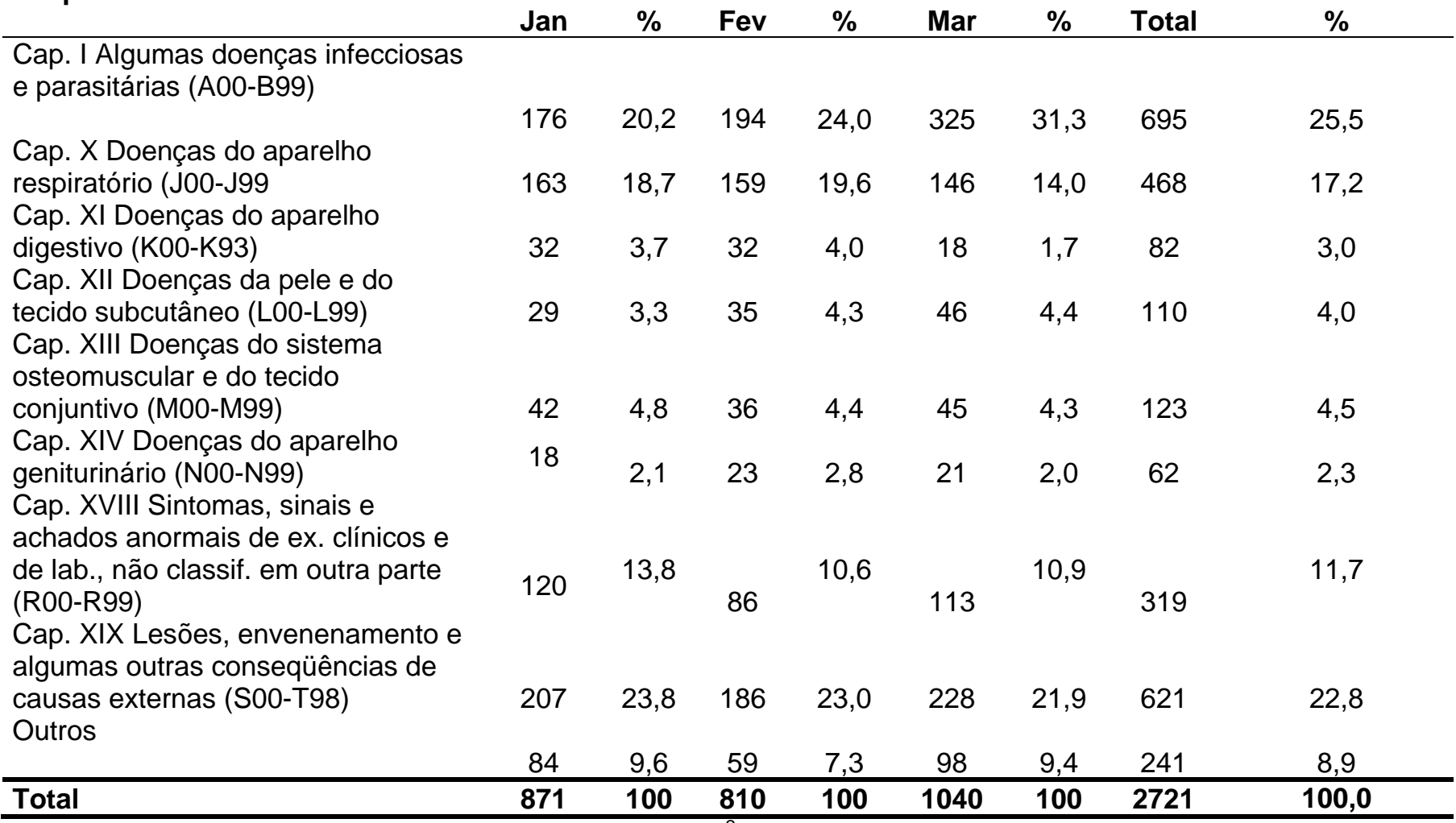

Significativo pelo teste de $\chi^{2}$, ao nível de $1 \%(p \leq 0,01)$.

No município de Londrina, nos meses de janeiro a março, ocorreram 984 internações entre as idades de 10 a 19 anos por gravidez, parto e puerpério $(29,8 \%)$ no sexo feminino, quanto que no sexo masculino foram lesões, envenenamento e algumas outras conseqüências de causas externas (12\%) (DATASUS, 2006),

Verificou-se no presente estudo que durante o mês de janeiro os maiores números de casos ocorreram por lesões, envenenamento e algumas conseqüências de causas externas, devido aos acidentes por fogos de artifício ocasionando queimaduras de vários graus e alguns acidentes devido ao abuso de bebidas alcoólicas (embriaguez), provavelmente por ser mês de férias e data festiva.

Segundo o período de atendimento e os grupos de causas da CID - 10, na Figura 1, verifica-se que a freqüência dos adolescentes no serviço ocorreu 
DUBUC, I. F.; FERRARI, R. A. P. Adolescentes atendidos num serviço público de urgência e emergência: perfil de morbidade e mortalidade. Revista Eletrônica de Enfermagem, v. 08, $\quad$. $\quad 02, \quad$ p. 250 - $258,2006 . \quad$ Disponível em http://www.fen.ufg.br/revista/revista8_2/v8n2a10.htm

principalmente no período da noite correspondendo $37,6 \%$ dos atendimentos, sendo importante informar que o período da noite compreende das 18 horas até
24 horas. No período da tarde ocorreram 31,8\% dos atendimentos, no horário da manhã, 24,5\%, enquanto que a madrugada apresentou $6 \%$.

Figura 1 - Distribuição dos adolescentes atendidos no pronto socorro de um hospital público segundo o período de atendimento, Londrina, janeiro a março, 2003.

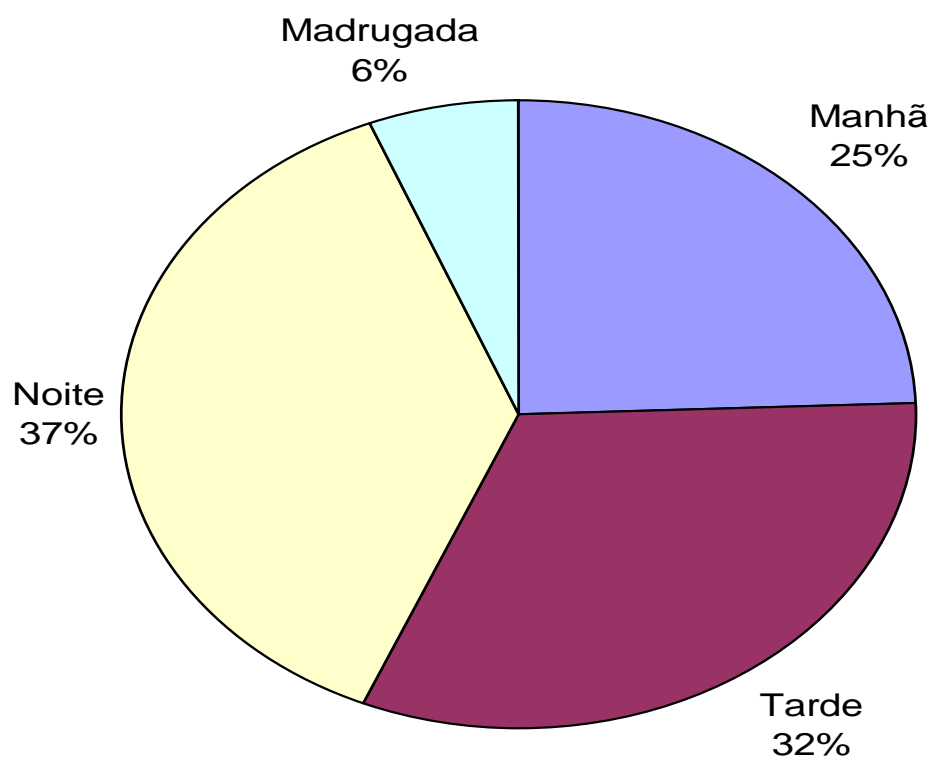

No estudo de YAMADA et al. (2002), os resultados demonstraram que as maiores ocorrências de atendimentos aos adolescentes foram no período noturno (22\%) por doenças respiratórias, diarréias e gastrenterites.

Nos períodos da tarde e da noite, destacou-se como primeira causa os atendimentos por doenças infecciosas e parasitárias seguida pelas lesões, envenenamento e algumas outras conseqüências de causas externas, enquanto que, no período da manhã prevaleceram as doenças infecciosas e parasitárias seguida pelas doenças do aparelho respiratório.

No período da noite ocorreu uma maior procura de pacientes por causas de doenças infecciosas e parasitárias, que em grande parte poderia ter sido atendido na unidade básica de saúde, visto que o horário de atendimento nas UBS é desde as sete horas da manhã até às dezenove horas.

Através da análise, verificou-se que o maior número de atendimento dos adolescentes foi realizado pela clínica médica (64\%), enquanto que a clínica ortopédica teve o menor número de atendimento (10\%).
De acordo com a rotina do HZN a especialidade de ortopedia faz plantão apenas uma vez por semana, e os casos com problemas ortopédicos depois de avaliados pelo clínico geral são encaminhados para outros hospitais de nível terciário.

$\mathrm{Na}$ Figura 2, pode-se observar que o maior número de atendimentos foram realizados pela clínica médica aos adolescentes com idade superior aos 12 anos, tendo como principais causas as infecções do aparelho respiratório - IVAS, viroses, gastrenterites e dengue. Com relação à clínica pediátrica, a maior freqüência de atendimento foi aos adolescentes menores de 12 anos (93,1\%), predominantes as IVAS, viroses e gastrenterites. A clínica cirúrgica atendeu todas as faixas etárias na mesma proporção, acima de $19 \%$, tendo como principais procedimentos as suturas de ferimentos cortes-contusos e curativos. Quanto à clínica ortopédica, também realizou atendimentos na mesma proporção em todas as idades, sendo as principais causas, de entorses e traumas. 
DUBUC, I. F.; FERRARI, R. A. P. Adolescentes atendidos num serviço público de urgência e emergência: perfil de morbidade e mortalidade. Revista Eletrônica de Enfermagem, v. 08, n. 02, p. 250 - $258,2006 . \quad$ Disponível em http://www.fen.ufg.br/revista/revista8_2/v8n2a10.htm

Figura 2 - Distribuição dos adolescentes atendidos no pronto socorro de um hospital público segundo a faixa etária e clínica, Londrina, janeiro a março, 2003

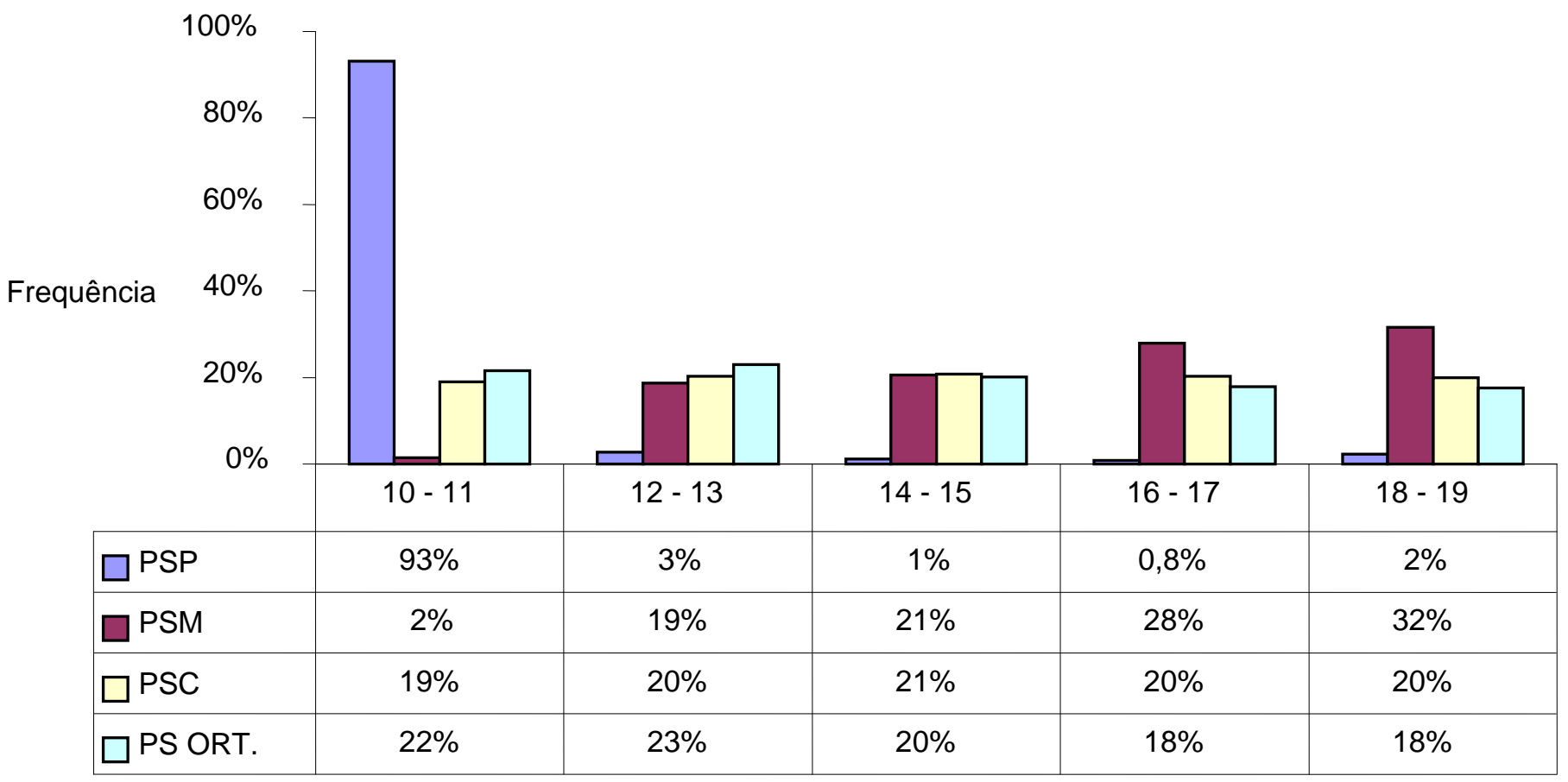

Em estudo realizado por CASTRO et al. (2002), verifica-se que em maiores de 15 anos a maior freqüência dos atendimentos foi da clínica médica, enquanto que a clínica pediátrica atendeu os adolescentes menores de 14 anos (44\%).

Cabe salientar, que $97 \%$ dos prontuários continham o diagnóstico, enquanto que os que não receberam diagnóstico se referem aos adolescentes que não aguardavam o atendimento médico.

$\mathrm{Na}$ Tabela 5, pode-se observar, que do total dos adolescentes atendidos nos meses de janeiro a março, 84,6\% receberam alta, 14\% foram transferidos e apenas 1,3\% (27) foram internados, estes em sua maioria para investigação diagnóstica do dengue, pneumonias e gastrenterites, entre outros.

Tabela 5 - Distribuição dos adolescentes atendidos no pronto socorro de um hospital público segundo a evolução, Londrina, janeiro a março, 2003.

\begin{tabular}{lrc}
\hline Evolução & $\mathbf{N}^{\circ}$ & $\%$ \\
\hline Alta & 1805 & 66,3 \\
\hline Evasão & 4 & 0,1 \\
\hline Transferência & 298 & 10,9 \\
\hline Internação & 27 & 1,0 \\
\hline Sem evolução & 588 & 21,6 \\
\hline Total & 2722 & 100,0 \\
\hline
\end{tabular}

Cabe salientar, dentre as causas que levaram os adolescentes a se evadirem do hospital foi a recusa da medicação prescrita após o atendimento médico.

Com relação às transferências realizadas pelo serviço, em sua maioria foi para serviços ambulatoriais e apenas uma foi encaminhada para um serviço hospitalar terciário do próprio município. Nenhum dos adolescentes atendidos no pronto socorro do hospital evolui para o óbito.

\section{CONSIDERAÇÕES FINAIS}

O estudo pretendeu trazer alguns conhecimentos sobre a morbidade por adolescentes atendidos em pronto socorro e por se tratar de um estudo transversal, as características apresentadas se referem a um momento específico, podendo sofrer modificações à medida que intervenções vão sendo implantadas. Neste sentido, ressalta-se a relevância do monitoramento das causas que levam os adolescentes a procurarem 0 serviço para a 
DUBUC, I. F.; FERRARI, R. A. P. Adolescentes atendidos num serviço público de urgência e emergência: perfil de morbidade e mortalidade. Revista Eletrônica de Enfermagem, v. $08, \quad$ n. $\quad 02, \quad$ p. $250 \quad-\quad 258,2006 . \quad$ Disponível em http://www.fen.ufg.br/revista/revista8_2/v8n2a10.htm

realização de novos estudos que aprofundem o conhecimento do tema e contribuam para a prevenção.

As informações coletadas não permitem avaliação da gravidade e evolução dos pacientes, visto que esses casos não são investigados de forma mais aprofundada e a grande maioria foi atendida apenas em ambulatório.

Observou-se que numa freqüência significativa durante a coleta de dados que as fichas de atendimentos não estavam preenchidas e quando preenchidas eram incompletas, principalmente pelo profissional médico.

Nesse sentido, cabe ao serviço de saúde promover sistematicamente condições para a execução do preenchimento dos dados da população atendida, pois é de grande relevância a fidedignidade destes para diminuir a subnotificação das informações para futuras pesquisas e intervenções de saúde.

\section{REFERÊNCIAS BIBLIOGRÁFICAS}

ANDRADE, S.M.; JORGE, M.H.P. de M. Características das vítimas por acidentes de transporte terrestre em município da Região Sul do Brasil. Rev. Saúde Pública, v.34 n.2, p.149-156, 2000.

AYRES, J.R.C.M.; FRANÇA JR., I. Saúde do adolescente. In: SCHRIBER, L.B.; NEMES, M.I.B.; GONÇALVES, R.B.M. (Orgs). Saúde do adulto: programa e ações na unidade básica. Ed. HUCITEC: São Paulo, 1996.

BAENINGER, R. Demografia da população jovem. In: SCHOR, N.; MOTA, M. do S.F. T.; BRANCO, V.C. (Orgs.). Cadernos juventude, saúde e desenvolvimento. Brasília: Ministério da Saúde, 1999.

BRASIL. Conselho Nacional de Saúde. Diretrizes e normas regulamentares de pesquisa envolvendo seres humanos. Brasília, DF, 1996. Resolução n 196 de 1996.

CAMACHO HUBNER, A.V. Perfil de salud sexual y reprodutiva de los y lãs adolescentes y jovens de América Latina y el Caribe: revision bibliográfica, 1988-1998. Washington, OPS, 2000.

CASTRO, C. J. et al (coords). Estudo do perfil da demanda do serviço de pronto socorro do Hospital Geral de Itaim Paulista. Säo Paulo; s.n; mar. 2002. 75 p. tab, graf.

DATASUS. Disponível em: http//www.datasus.gov.br [Acesso em 14 de fev. 2006].

INSTITUTO BRASILEIRO DE GEOGRAFIA E ESTATÍSTICA. Censo demográfico 2000: resultando do universo. Disponível em: http://www.ibge.gov.br/censo/. [Acesso em 20 de jan. 2006].
LYRA, J. et al. "A gente não pode fazer nada, só podemos decidir sabor de sorvete". Adolescentes: de sujeito de necessidades a um sujeito de direitos. Cad. CEDES, v.22, n.57, p.9-21, 2002.

LONDRINA. Secretaria Municipal de Planejamento. Síntese do perfil do município de Londrina 2003: baseado nos dados de 2002. Londrina, 2003. Disponível

em:

http://www.londrina.pr.gov.br/planejamento/perfil/2003 /perfil2003.pdf. [Acesso em 10 fev. 2006].

LONDRINA. Secretaria Municipal de Saúde. Diretoria de Assistência à Saúde. Dados do SIAB - Londrina 2005. Londrina, 2006.

MONTEIRO, S.; VARGAS, E.P. e REBELLO, S.M. Educação, prevenção e drogas: resultados e desdobramentos da avaliação de um jogo educativo. Educ. Soc., v.24, n.83, p.659-678, 2003.

ORGANIZAÇÃO MUNDIAL DE SAÚDE. Classificação Estatística Internacional de Doenças e Problemas Relacionados à Saúde. Décima Revisão (CID - 10). Centro Colaborador da OMS para a Classificação de Doenças em Português. $8^{a}$ ed. $10^{a}$ revisão - São Paulo: Editora da Universidade de São Paulo (EDUSP), 2000.

ORGANIZACIÓN MUNDIAL DE LA SALUD. La salud de los jovens: Um reto y uma esperanza. Genebra, 1995.

RIOS, L.F.et al. Rumo à adultez: oportunidades e barreiras para a saúde sexual dos jovens brasileiros. Cad. CEDES, v.22, n.57, p.45-61, 2002.

TAKEMURA, N.S. \& ANDRADE, S. M. Dengue em Londrina, Paraná: conhecimento sobre a doença e adesão a medidas preventivas. In: VIII Congresso Paulista de Saúde Pública, 2003, Ribeirão Preto (SP). YAMADA, A. T. T. et al (coords). Estudo do perfil da demanda do serviço de pronto socorro do hospital geral de Itaquaquecetuba. São Paulo; S.n ; agosto/2002

Artigo original recebido em 20.09.2006 Aprovado para publicação em 30.09.2006 